\title{
Neutrophil extracellular traps induced by VP1 contribute to pulmonary edema during EV71 infection
}

\author{
Nan Wang ${ }^{1,2,3}$, Xiaofan Yang ${ }^{4}$, Jiandong Sun ${ }^{5}$, Zhixiao Sun ${ }^{3}$, Qiyun $\mathrm{Ma}^{3}$, Zhengxia Wang ${ }^{3}$, Zhongqi Chen ${ }^{3}$, \\ Zibin Wang ${ }^{6}$, Fan $\mathrm{Hu}^{7}$, Huijuan Wang ${ }^{1}$, Linfu Zhou ${ }^{3}$, Mingshun Zhang $\mathbb{1}^{1,2}$ and Juan $\mathrm{Xu}^{1}$
}

\begin{abstract}
Pulmonary edema is a fatal complication of EV71-associated hand, foot, and mouth disease (HFMD). The pathogenesis of EV71-induced pulmonary edema remains largely unclear. In this study, we aimed to explore the roles of the capsid protein VP1 in the occurrence of EV71-induced pulmonary edema. The intranasal inoculation of recombinant VP1 protein caused lung inflammation with an elevation of inflammatory cytokines and neutrophils infiltration. Moreover, neutrophil extracellular traps (NETs) were observed in the lung parenchyma of the mice treated with VP1. VP1 directly induced the formation of NETs, which depended on PAD4. VP1 also damaged the lung barrier via the reduction of the tight junction protein occludin. Moreover, the EV71 attachment receptor vimentin was increased upon VP1 administration. In contrast, NETs decreased vimentin levels, suggesting a novel role for NETs in viral immune defense. These results evidenced a direct role of VP1 in EV71-induced pulmonary edema and demonstrated that NETs may be both harmful and beneficial in EV71 infection.
\end{abstract}

\section{Introduction}

Hand, foot, and mouth disease (HFMD) is an infectious disease that affects millions of young children and infants worldwide ${ }^{1}$. Enterovirus 71 (EV71) is considered as the major cause of HFMD. Although most HFMD is selflimiting and is a mild illness, EV71 infection may cause serious neurological, cardiac, and respiratory problems in young children ${ }^{2}$. Pulmonary edema is considered as the major cause of HFMD-related fatalities. It is postulated that the occurrence of pulmonary edema is associated with CNS lesions ${ }^{3}$. However, the pathogenesis of EV71induced pulmonary edema is still largely unclear.

\footnotetext{
Correspondence: Mingshun Zhang (mingshunzhang@njmu.edu.cn) or Juan Xu (xiangbangbang@njmu.edu.cn)

'Department of Immunology, Nanjing Medical University, 210016 Nanjing, China

${ }^{2} \mathrm{NHC}$ Key Lab of Antibody Technique, Nanjing Medical University, 210016 Nanjing, China

Full list of author information is available at the end of the article.

These authors contributed equally: Nan Wang, Xiaofan Yang, Jiandong Sun

Edited by A. Rufini
}

In a previous study, EV71 mRNAs were detected in lung tissue, providing evidence for EV71 replication in lung tissue $^{4}$. Further, EV71 antigen was widely distributed in lung tissue both in fatal EV71 patients and in an EV71infected mouse model ${ }^{5}$. VP1 is acapsid protein responsible for EV71 genotyping ${ }^{6}$ and virus entry ${ }^{7}$. We hypothesize that the presence of VP1 in the lung tissue could be involved in lung injury. Therefore, we directly introduced recombinant VP1 protein into the lungs of mice and examined the inflammatory responses.

Neutrophil extracellular traps (NETs) ${ }^{8}$ are composed of nuclear chromatin, hypercitrullinated histones, granular antimicrobial proteins, and cytoplasmic proteins ${ }^{9}$. Similar to a double-edged sword, NETs may function in immune defenses, but they may also magnify inflammation ${ }^{10}$ in diverse diseases. Many types of viruses can induce NET formation ${ }^{11-13}$, and recent studies have revealed that excessive NET formation may cause adverse effects in a number of lung diseases, including acute lung injury ${ }^{14-16}$.

\section{(c) The Author(s) 2019}

(c) (i) Open Access This article is licensed under a Creative Commons Attribution 4.0 International License, which permits use, sharing, adaptation, distribution and reproduction cc) in any medium or format, as long as you give appropriate credit to the original author(s) and the source, provide a link to the Creative Commons license, and indicate if changes were made. The images or other third party material in this article are included in the article's Creative Commons license, unless indicated otherwise in a credit line to the material. If material is not included in the article's Creative Commons license and your intended use is not permitted by statutory regulation or exceeds the permitted use, you will need to obtain permission directly from the copyright holder. To view a copy of this license, visit http://creativecommons.org/licenses/by/4.0/. 
We further evaluated whether VP1 could induce the formation of NETs in the lung.

Moreover, life-threatening pulmonary edema may be accompanied by the loss of epithelial integrity. Tight junctions and adherens junctions have barrier functions. ZO-1 and occludin are two of the best-characterized tight junction proteins ${ }^{17-19}$. In addition, E-cadherin is an important adherens junction protein ${ }^{20}$. Therefore, we explored the role of VP1 in the alterations of the lung epithelial barrier and junction proteins. The intermediate filament protein vimentin is a type of adherens junction protein. More importantly, vimentin contributes to EV71 virus entry ${ }^{21}$. Finally, we examined the roles of VP1 and NETs in the expression of vimentin.

\section{Results}

\section{VP1-induced acute lung injury}

We introduced VP1 directly into mouse lung tissue by intranasal administration to evaluate whether VP1 could induce acute lung injury. Our study revealed that the wetto-dry (W/D) ratio in the VP1 group was significantly higher than that of the blank and NS groups (Fig. 1a), which was indicative of pulmonary edema and of injury to the pulmonary epithelial barrier. Accordingly, the transcript levels of inflammatory cytokines (TNF- $\alpha$, IL-1 $\beta$, and IL-6) in mice treated with VP1 were significantly increased compared to the control groups (Fig. 1b). Moreover, hematoxylin and eosin (H\&E) staining suggested that there was destruction in pulmonary epithelial architecture, an increase in inflammatory cell infiltration in the alveolar space and an increase in interstitial edema in the VP1-treated mice (Fig. 1c). In brief, these observations demonstrated that more severe lung injury occurred in the mice with VP1 exposure compared with that in the blank and saline groups.

\section{VP1 recruited neutrophils into the lung}

Further, we analyzed the types of inflammatory cells that had infiltrated into lung tissue after VP1 inhalation using flow cytometry. As shown in Fig. 2a, b, neutrophil infiltration increased in the $1 \mu \mathrm{g}$ VP1-treated group compared to the saline group. Although VP1 caused an increase in macrophages compared with the control group, it was not statistically significant (Fig. 2c, d). In conclusion, VP1 promoted neutrophil infiltration into lung tissue.

\section{VP1 increased neutrophil chemokines in lung tissue}

To further clarify the reasons for the increased neutrophils and macrophages infiltration, we examined neutrophil chemokines (KC, CXCL2, and CXCL5) $)^{22-24}$ and macrophage chemokines (MCP-1, CCL3, and CCL4) ${ }^{25,26}$ in lung from the mice that were or were not treated with VP1. As shown, the transcript levels of the neutrophil chemokines KC (Fig. 3a), CXCL2 (Fig. 3b), and CXCL5 (Fig. 3c) and the macrophage chemokines MCP-1 (Fig. 3e), CCL3

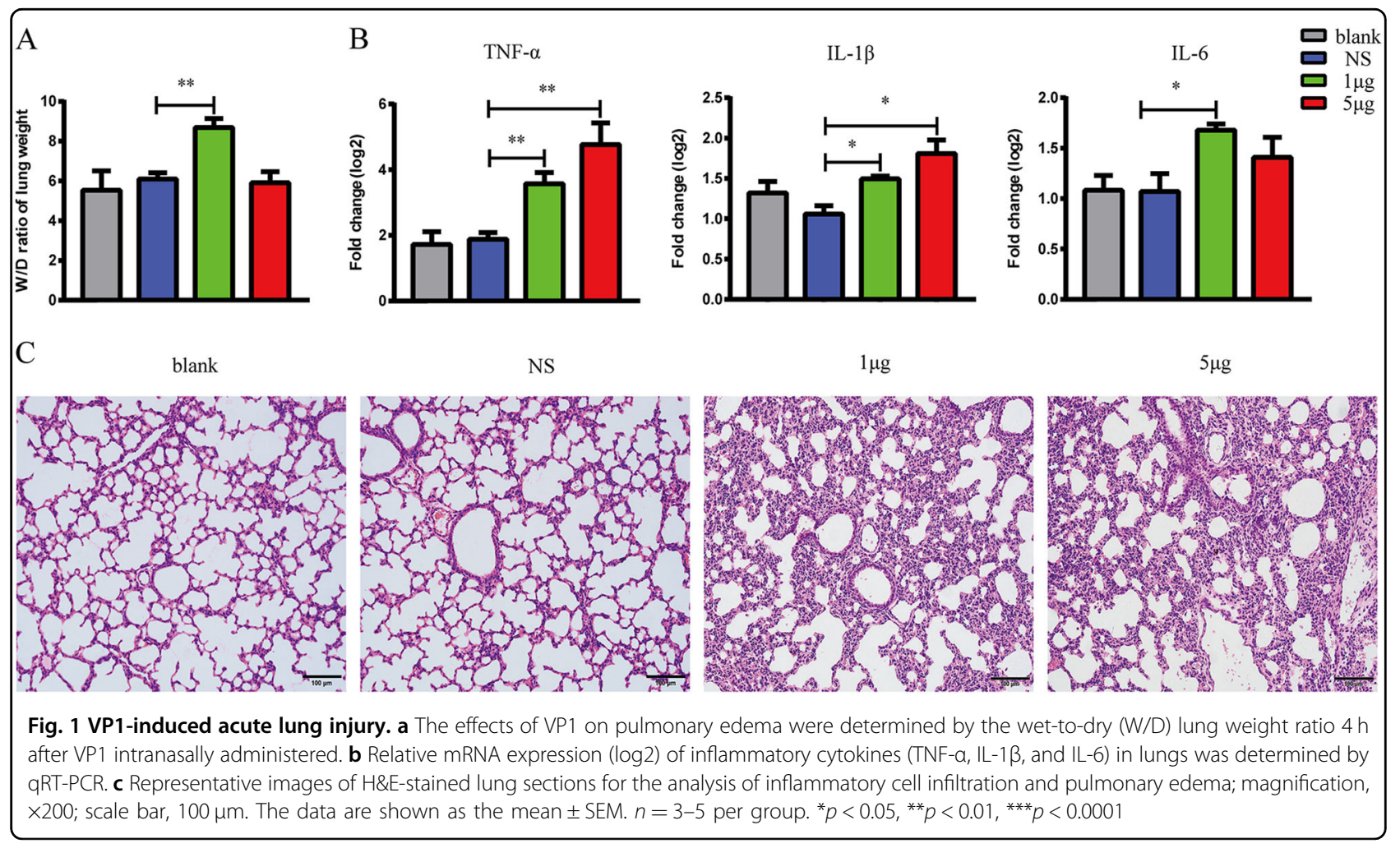



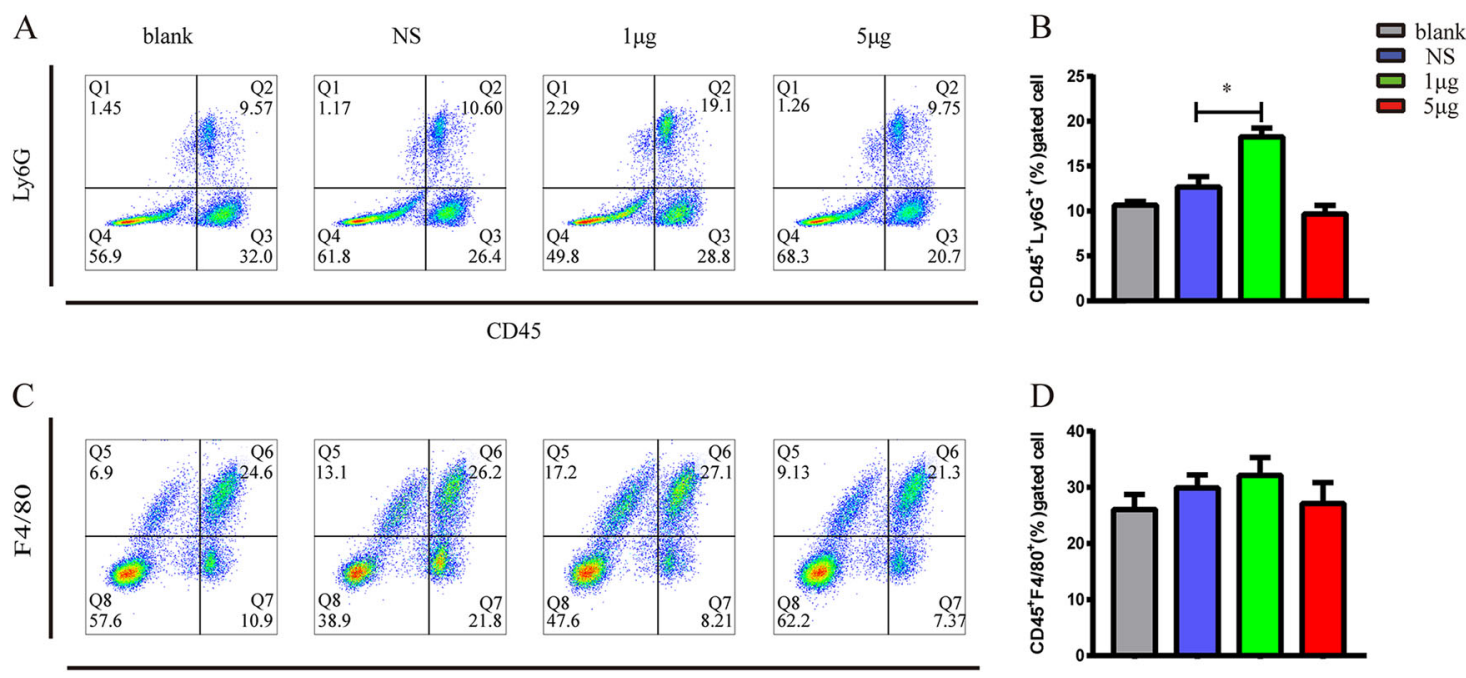

$\mathrm{CD} 45$

Fig. 2 VP1 recruited neutrophils into the lung. The single-cell suspensions were obtained from the lungs of mice from each experimental group and stained with anti-CD45-FITC, anti-Ly6G-PE, and anti-F4/80-APC antibodies. Flow cytometric analysis was performed and representative flow cytometry pseudocolor plots gated on neutrophils $\left(\mathrm{CD} 45^{+} \mathrm{Ly} 6 \mathrm{G}^{+}\right)$and macrophages $\left(\mathrm{CD} 45^{+} \mathrm{F} 4 / 80^{+}\right)$were shown. $\mathbf{a}$, $\mathbf{b}$ The percentage of neutrophils in the lung was markedly increased in VP1-treated mice. $\mathbf{c}$, $\mathbf{d}$ The percentage of macrophages in the lung was slightly increased in VP1-treated mice. Values are expressed as the mean \pm SEM. $n=4-6$ per group. ${ }^{*} p<0.05,{ }^{* *} p<0.01,{ }^{* *} p<0.0001$

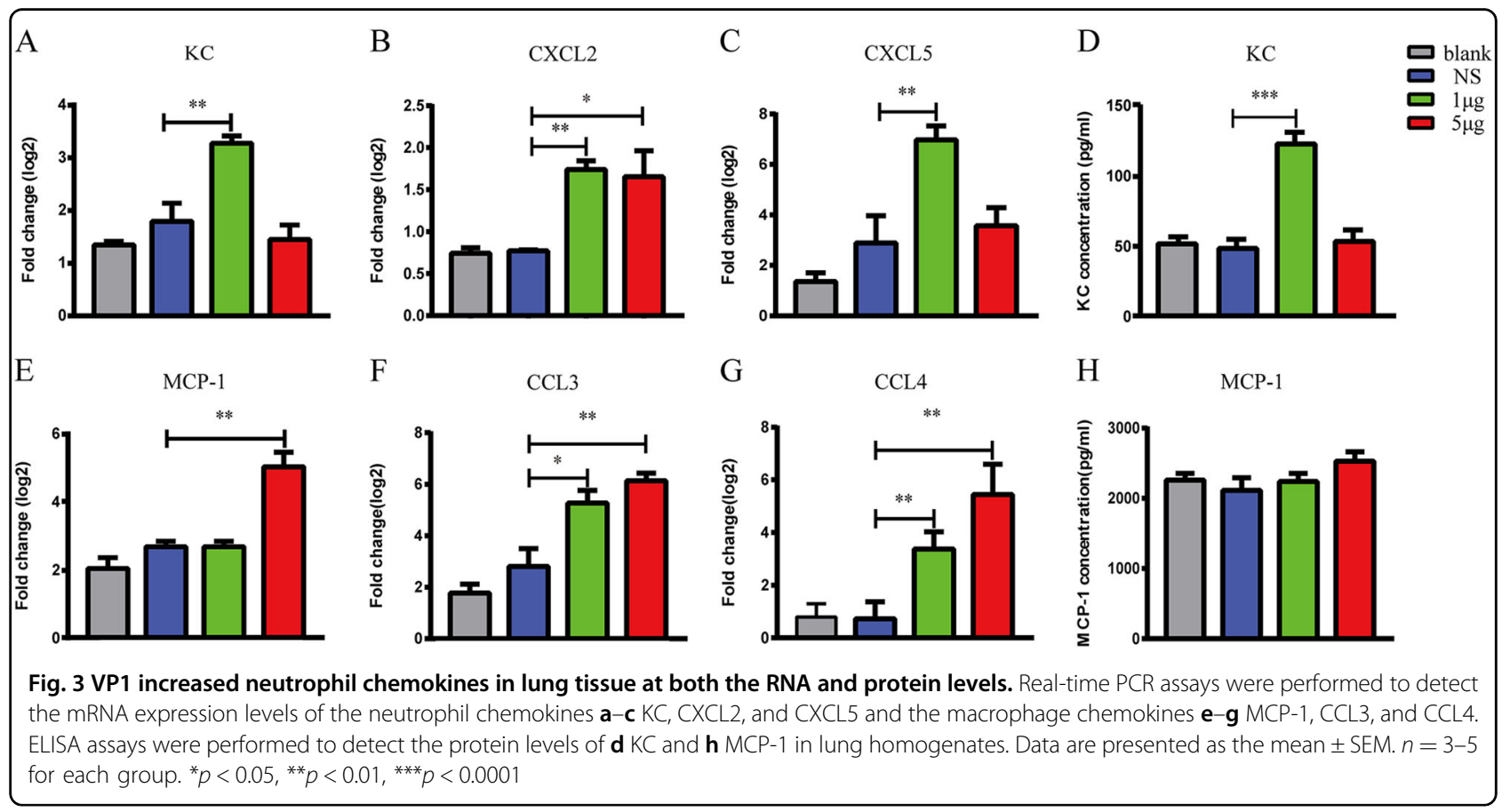

(Fig. 3f), and CCL4 (Fig. 3g) were significantly increased in the VP1-treated mice compared with that in the blank and saline groups. Furthermore, VP1 increased the KC protein expression in the lung homogenates (Fig. 3d). Consistent with the results shown in (Fig. 2d), there was no significant increase in the macrophage chemokines MCP-1 (Fig. 3h) in the VP1-treated group. In summary, neutrophils infiltrated into lung tissue in VP1-treated mice may at least partly depend on chemokines.

\section{VP1-induced neutrophil extracellular traps}

We further explored whether VP1 could induce NET formation and subsequently lead to acute lung injury. To test this hypothesis, we detected citrullinated histones 


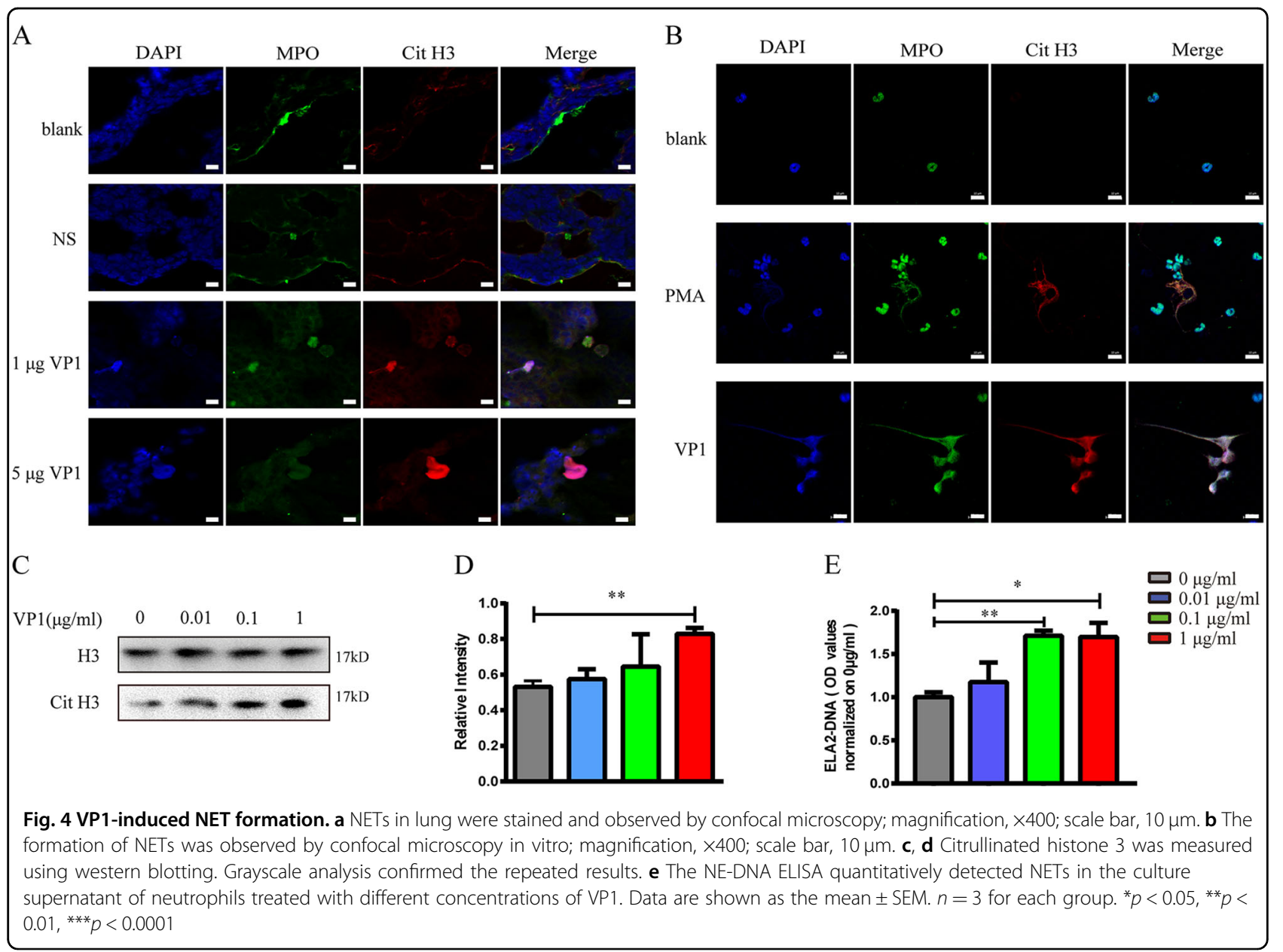

and myeloperoxidase (MPO) in lung tissues of each treatment group by immunofluorescence. As expected, NETs were detected in lung tissue of mice treated with VP1, which was demonstrated by the presence of extracellular DNA overlaid with citrullinated histone 3 and MPO (Fig. 4a). To determine whether VP1 may directly induce NETs, neutrophils that were isolated from the bone marrow of mice were stimulated with different concentrations of VP1. As shown in Fig. 4e, neutrophil elastase-DNA (NE-DNA) complex expression was significantly elevated in a concentration-dependent manner in the culture supernatant of neutrophils treated with VP1. Similarly, western blotting showed that citrullinated histone 3 also increased in a concentrationdependent manner (Fig. 4c, d). According to the NEDNA ELISA and western blotting results shown above, $1 \mu \mathrm{g} / \mathrm{ml}$ VP1 was selected to stimulate NET formation, and NET formation was also observed by confocal microscopy; this was similar to stimulation by $100 \mathrm{nM}$ phorbol-myristate acetate (PMA), which is commonly employed as a potent nonphysiologic agonist to induce NET formation in vitro ${ }^{27,28}$ (Fig. 4b). Collectively, VP1 directly promoted the formation of NETs that may further damage lung tissue.

\section{Dynamic observation of NET formation by a living-cell imaging technique}

To more directly observe NET formation, neutrophils with or without VP1 stimulation were placed into a livecell imaging system for real-time observation ${ }^{29,30}$. SYTOX orange $(1 \mu \mathrm{M})$ was added to each cell culture well before neutrophils were placed into a live-cell imaging system ${ }^{31}$. Images were acquired with a Cell Discoverer 7 live-cell imaging system (Carl Zeiss Microscopy) using an excitation wavelength of $558 \mathrm{~nm}$, and detection was performed using an emission wavelength of $575 \mathrm{~nm}$. We observed that NETs formed in the VP1-treated neutrophils (Fig. 5). In addition, NETs were first observed at $\sim 90 \mathrm{~min}$ after VP1 stimulation (refer to the video in the supplementary materials for details $)^{32}$.

\section{VP1 promotes NETosis via a PAD4-dependent pathway}

The peptidylarginine deaminase 4 (PAD4)-dependent histone citrullination and decondensation of chromatin 

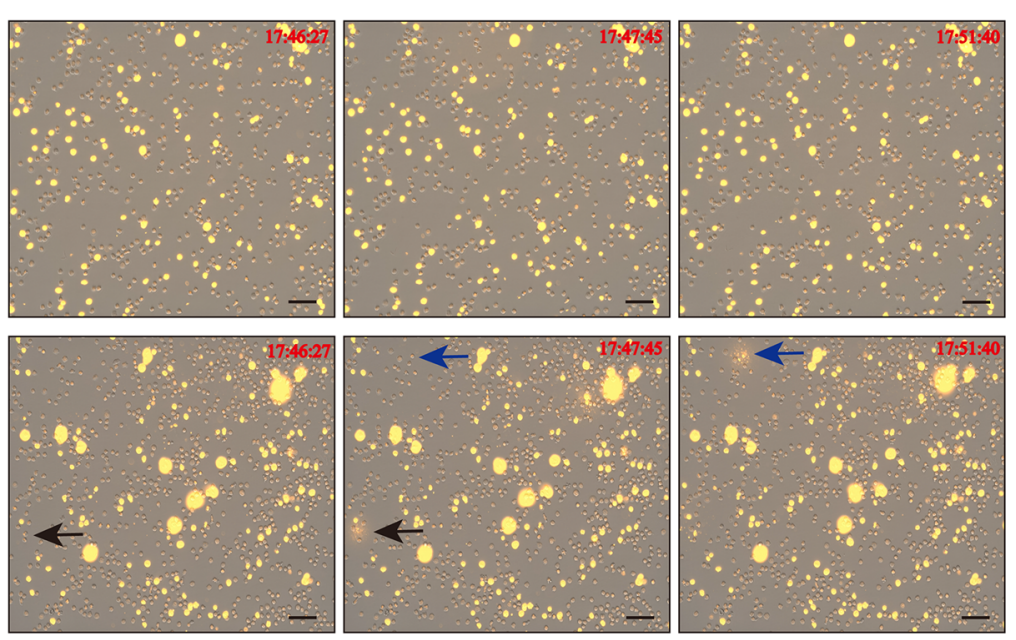

control

Fig. 5 Dynamic observation of NET formation by a living-cell imaging technique. The time in the picture referred to the time when the data were collected by a Zeiss Cell Discoverer 7 microscope system; scale bar, $50 \mu \mathrm{m}$. Two arrows (black and blue) indicated the neutrophils undergoing NETS

have been regarded as a key step in the formation of $\mathrm{NETs}^{33-35}$. NETosis also depends on the generation of reactive oxygen species by NADPH oxidase ${ }^{36,37}$. However, an understanding of the effect of these enzymes on VP1 remains lacking. We used citrullinated histone 3 as a biomarker for neutrophil priming for NETosis, and we examined the levels of circulating $\mathrm{CitH}^{+}$cells and found that a greater percentage of neutrophils was primed for NETosis in the VP1-treated mice (Fig. 6a), which was second only to the levels observed in the positive control (PMA) group. To further evaluate how VP1 triggered NETosis, we added the PAD4 inhibitor $\mathrm{Cl}$-amidine ${ }^{38,39}$ or the NADPH oxidase inhibitor diphenyleneiodonium $(\mathrm{DPI})^{40,41}$ half an hour before VP1 stimulation. We found that $\mathrm{Cl}$-amidine inhibited NET formation triggered by VP1 (Fig. 6b, c), whereas DPI did not (data not shown). In conclusion, VP1 promoted NET formation in a PAD4dependent and NADPH oxidase-independent manner.

\section{VP1 directly damages lung tissue barrier function}

Previous results confirmed that VP1 promoted the infiltration of neutrophils into lung tissue by increasing the chemokine levels of neutrophils and indirectly damaged lung tissue by promoting the formation of NETs. Next, we explored whether VP1 could directly damage the pulmonary epithelial barrier function both in vivo and in vitro. The bronchoalveolar epithelial permeability was determined by measuring the leakage of FD4 from the blood vessels into the lung. As shown in Fig. $7 \mathrm{a}$, the fluorescence density of VP1-treated mice was significantly higher than that of the blank group and normal saline group. Since VP1 treatment increased the bronchoalveolar epithelial permeability in mice, it was likely to change the morphology of the junctions in the alveolar epithelium. The integrity of the pulmonary epithelium barriers and the morphological alterations of the junctions were studied by transmission electron microscopy in mice with or without VP1 treatment. The random sampling of the left lung from the blank and NS groups showed intact junctions between all of the epithelial cells. In contrast, after exposure to VP1 for $4 \mathrm{~h}$, the random sampling of the left lungs from VP1-treated mice showed the loss of junctions between the alveolar epithelial cells. The junctions were irregularly widened (Fig. 7b). The protein expression levels of occludin were decreased in the VP1 group compared to the blank and NS groups. In contrast, there was no significant change in the protein levels of ZO-1 and E-cadherin in each treatment group (Fig. 7c). In vitro transepithelial electrical resistance (TEER) measurements were carried out to evaluate the integrity of the MLE-12 cellular monolayers $^{42,43}$. VP1 significantly decreased the TEER and epithelial integrity of the pulmonary epithelial cells. (Fig. 7d). Collectively, VP1 may damage the pulmonary epithelial barrier function via the downregulation of tight junction protein occludin.

\section{VP1 increased vimentin, while NETs downregulated vimentin on the pulmonary epithelial cells}

The junction protein vimentin is an attachment receptor for EV71 ${ }^{21}$. Intranasal introduction of VP1 increased the expression of vimentin in lung tissue (Fig. 8a). To further explore whether vimentin could be augmented on the lung epithelial cells by VP1, MLE-12 cells were stimulated with recombinant VP1 protein. As shown in Fig. $8 \mathrm{~b}-\mathrm{d}$, VP1 increased vimentin expression in the lung epithelial cells. Interestingly, vimentin was downregulated when NETs acted directly on the MLE-12 cells (Fig. 8e). 

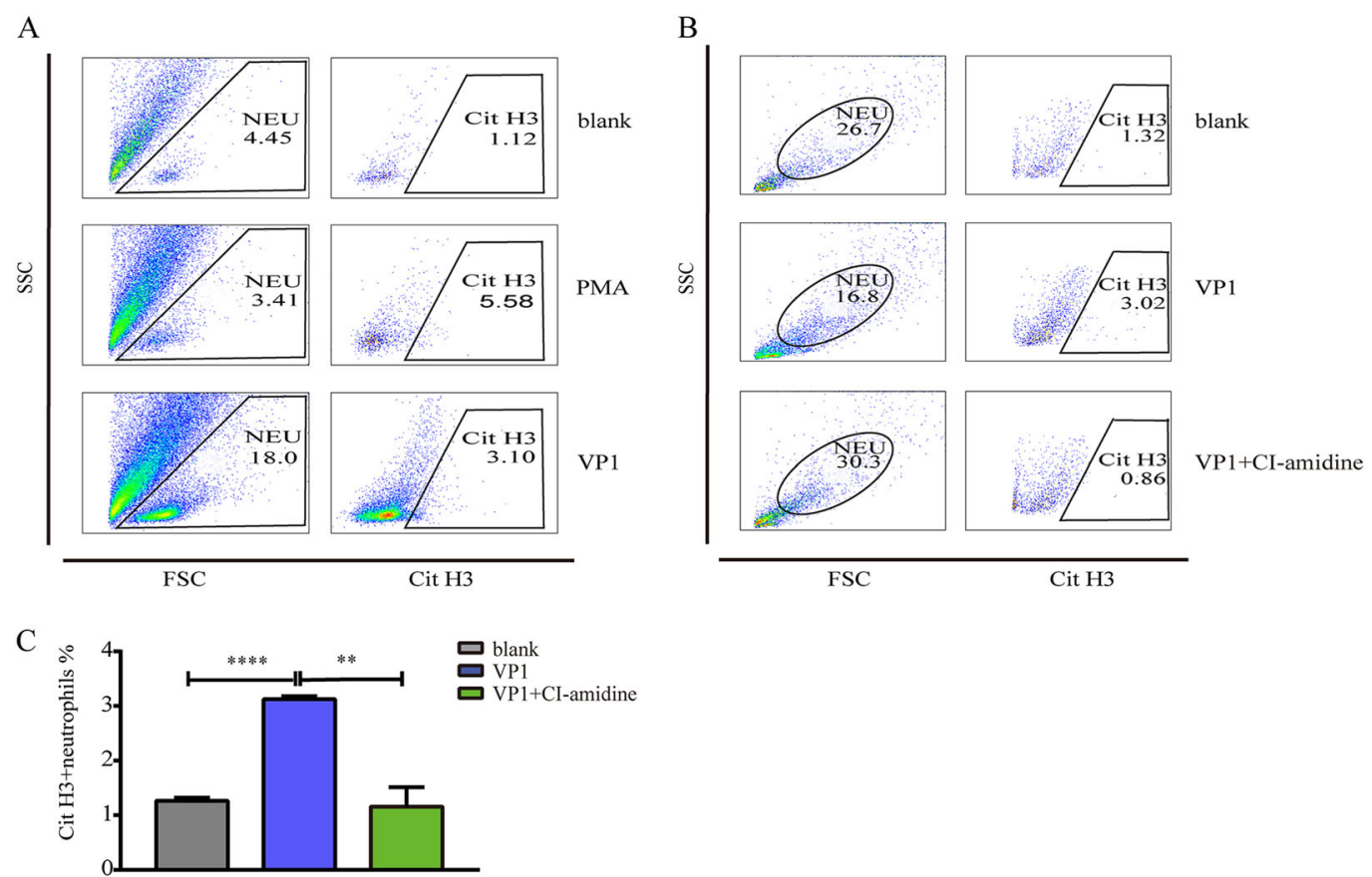

Fig. 6 VP1 promoted NETs via the PAD4-dependent pathway. a Flow cytometry analysis revealed the percentage of citrullinated H3-positive neutrophils. b, c The PAD4 inhibitor Cl-amidine significantly decreased the percentage of citrullinated H3-positive neutrophils in the VP1-treated group. Data are shown as the mean \pm SEM. $n=3$ for each group. ${ }^{*} p<0.05,{ }^{* *} p<0.01,{ }^{* * *} p<0.0001$

Of note, vimetin in the control group without stimulus seemed increased during the time course (Fig. 8c, e), which was in the line with the observation that epithelial cells growth and migration may cause the elevation of vimetin $^{44}$. In summary, VP1 may positively promote the virus receptor vimentin, which was negatively regulated by NETs.

\section{Discussion}

Pulmonary edema is a major cause of EV71-associated mortality. Some studies have shown that compared with EV71 patients with uncomplicated brainstem encephalitis, patients with brainstem encephalitis accompanied by pulmonary edema have significantly higher serum and CSF levels of proinflammatory cytokines ${ }^{45,46}$. In addition, inflammatory cells (neutrophils, macrophages, mast cells, etc.) were demonstrated to contribute to EV71 pulmonary edema $^{4,47}$. VP1 is the major virulence determinant of EV71. However, the pathological roles of VP1 in the initiation and progression of pulmonary edema remain largely elusive. Intranasal inoculation of recombinant VP1 protein promoted lung inflammation that was characterized by elevated inflammatory cytokines (TNF- $\alpha$, IL- $1 \beta$, and IL-6) and by neutrophils in the lung parenchyma. The increase in the chemokine $\mathrm{KC}$ may promote neutrophil infiltration into the lung, which was in accordance with previous findings that showed that neutrophils were widely present in the tissues and organs of patients infected with EV71 ${ }^{4}$. Of note, VP1 at $1 \mu \mathrm{g}$ seemed more potent that VP1 at $5 \mu \mathrm{g}$ in the mouse model of acute lung injury, we observed that $5 \mu \mathrm{g}$ VP1 diluted in $10 \mu \mathrm{l}$ NS was a little viscous, which may cause the inadequate inhalation in the intranasal instillation and the consequently reduced effects. Still, our observations suggested that pulmonary edema might be the result not only of the direct hydrostatic force of systemic vasoconstriction resulting from brainstem injury but also of an increased pulmonary inflammatory response.

Although they were first identified in bacterial infections, NETs are also involved in viral infections ${ }^{48}$. The roles of NETs in EV71 infection, however, have not been demonstrated before. In the lung parenchyma of VP1treated mice, we observed the formation of NETs. Interestingly, VP1 may directly trigger the formation of NETs, which was at least partially dependent on PAD4. Considering that different stimuli, including inflammatory cytokines, may trigger the formation of NETs, we could not preclude the possibility that EV71 may directly (via VP1) or indirectly (via inflammatory cytokines) promote the formation of NETs.

Damage to pulmonary permeability may cause fatal lung edema. As the W/D ratio increased and the fluorescence dextran leakage increased in the VP1-treated lung parenchyma, the pulmonary permeability became damaged, 
A

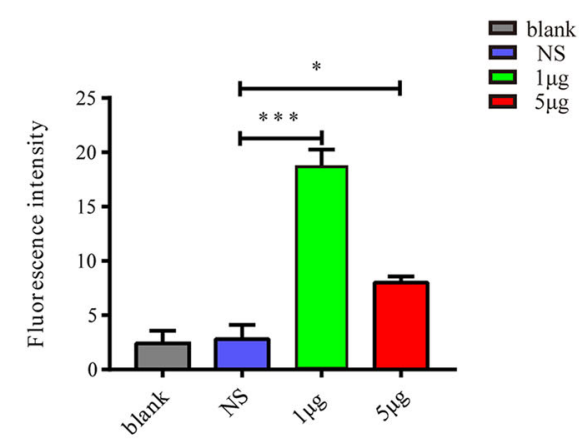

$\mathrm{C}$

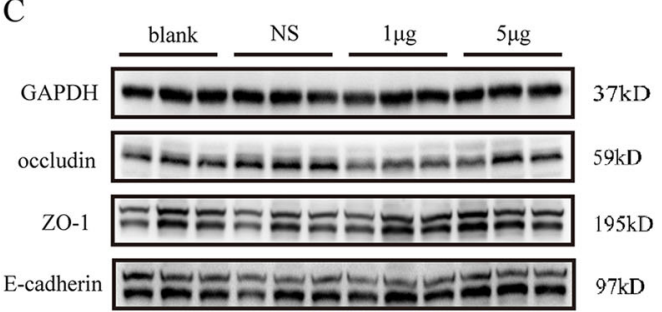

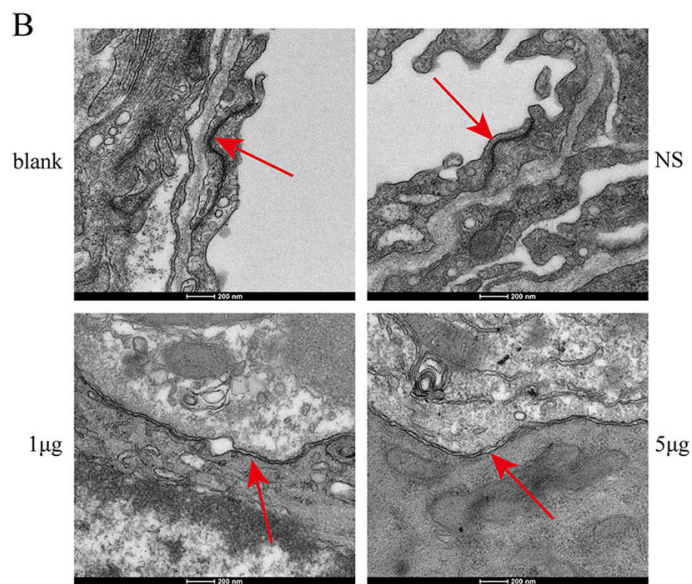

$\mathrm{D}$

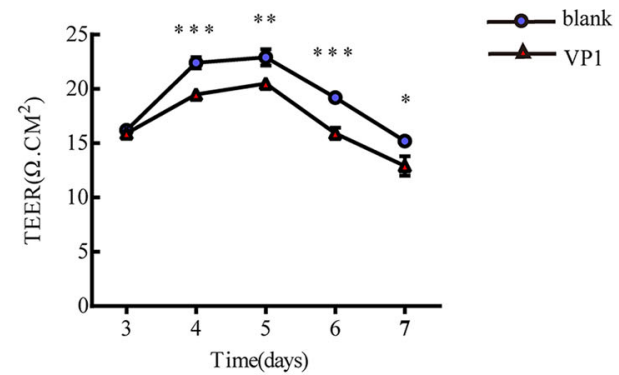

Fig. 7 VP1 directly damaged lung tissue barrier function. a Bronchoalveolar epithelial permeability was determined by measuring the leakage of FD4 in lung. Data are presented as the mean \pm SEM. $n=3-5$ for each group. $\mathbf{b}$ The integrity of the pulmonary epithelium and morphological alterations of junctions was studied by transmission electron microscopy in both VP1-treated and untreated groups. $\mathbf{c}$ The western blotting assay of lung tissue tight junction protein and adherens junction protein in each group. $\mathbf{d}$ The TEER of the MLE-12 cellular monolayer over time treated or untreated with VP1. Data are expressed as the mean \pm SEM, $n=3$. ${ }^{*} p<0.05$, ${ }^{* *} p<0.01$, ${ }^{* * *} p<0.0001$

which was directly observed using transmission electron microscopy. The tight junction protein occludin was involved with lung integrity, and an occludin reduction may be accompanied by lung edema ${ }^{49}$. Accordingly, occludin was significantly decreased in the lungs of VP1treated mice. In contrast to the tight junction protein occludin, the adherens junction protein vimentin mediates EV71 virus entry ${ }^{21}$. VP1 increased the expression of vimentin, suggesting that EV71 may amplify the infection with the increase in the virus receptor. Interestingly, VP1induced NETs caused the downregulation of vimentin and, therefore, may alleviate EV71 infection. Therefore, NETs may not only damage the lung barriers as observed in the acute lung injury induced by other stimuli but may also contribute to the immune defense via the downregulation of virus receptors. Recently, it was shown that NETs may awaken dormant cancer cells via the cleavage of matrix proteins ${ }^{50}$. The mechanisms of NETs degrading vimentin warrant further study.

In conclusion, our study revealed that the EV71 capsid protein VP1 directly caused neutrophil infiltration and NET formation, which damaged the lung barrier and caused pulmonary edema. Moreover, NETs reduced expression of the virus receptor vimentin, which may represent a novel function of NETs in the viral immune defense.

\section{Materials and methods}

\section{Animals and ethics statement}

Wild type C57BL/6J mice (1 week old) that were maintained under specific pathogen-free conditions were obtained from the College of Veterinary Medicine of Yangzhou University (Yangzhou, China). The room temperature and humidity were set at $23 \pm 3^{\circ} \mathrm{C}$ and $55.5 \pm 10 \%$, respectively. All mice were maintained on a 12-h light-dark cycle. All animal protocols were reviewed and approved by the Animal Care and Use Committee of the Nanjing Medical University (1708004).

\section{VP1-induced acute lung injury}

VP1 recombinant protein with low endotoxin $(<0.1 \mathrm{EU} /$ $\mu \mathrm{g})$ was obtained from Genscript (Nanjing, China). Experimental animals were randomly divided into four groups: the blank control group (blank), saline group (NS), $1 \mu \mathrm{g}$ VP1 group $(1 \mu \mathrm{g})$, and $5 \mu \mathrm{g}$ VP1 group $(5 \mu \mathrm{g})$. The blank control group was untreated. The NS and VP1 


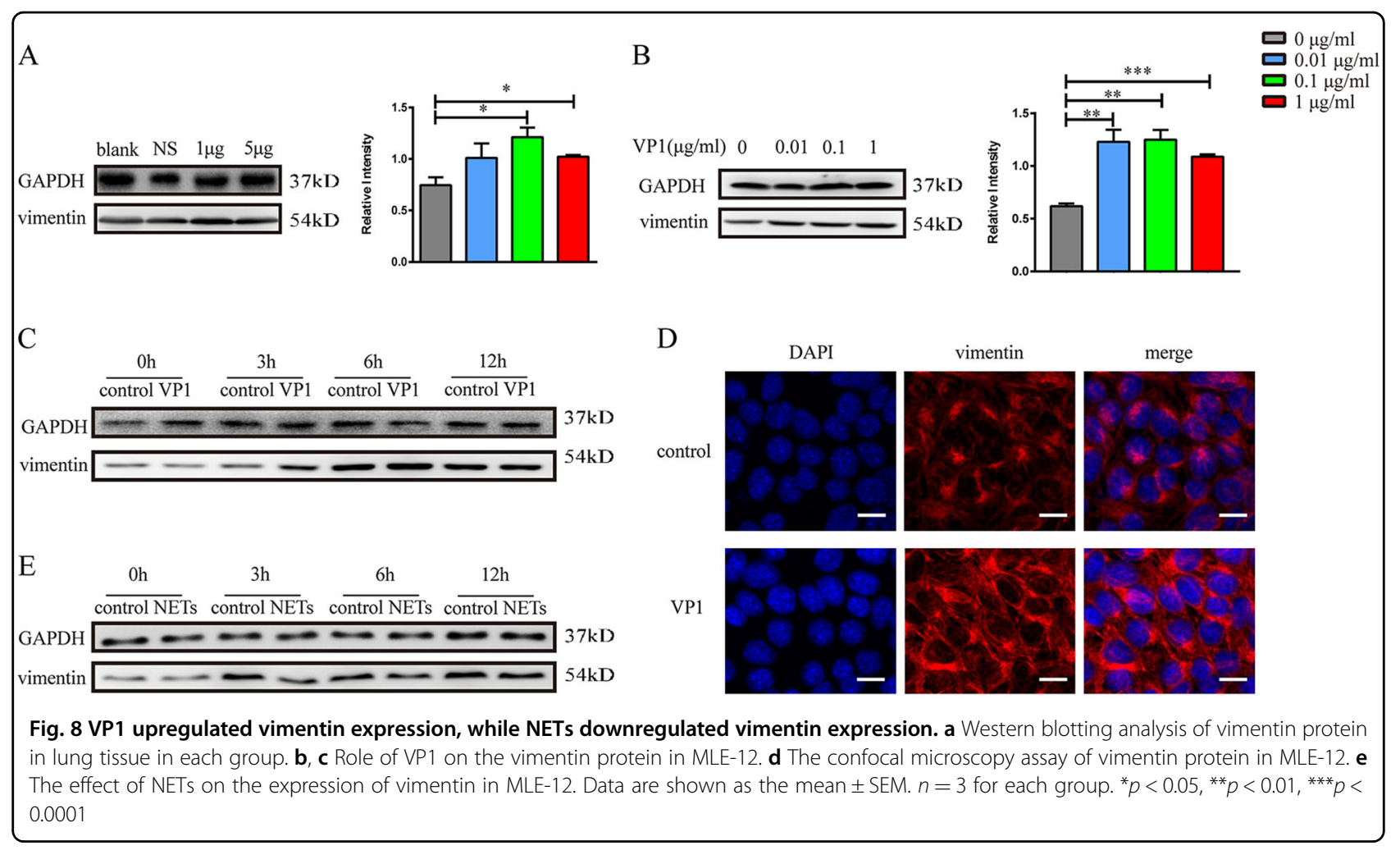

groups were given NS droplets or NS droplets containing VP1 $(1 \mu \mathrm{g}$ or $5 \mu \mathrm{g}$ of protein diluted in $10 \mu \mathrm{l}$ of NS) intranasally after anesthetization by intraperitoneal injection with a mixture of $10 \mathrm{mg} / \mathrm{kg}$ xylazine (MTC Pharmaceuticals, Cambridge, ON, Canada) and $200 \mathrm{mg} / \mathrm{kg}$ ketamine hydrochloride (Rogar/STB, London, ON, Canada).

\section{Lung histopathological examination}

Four hours after the last inoculation with saline or VP1, PBS was pumped into the right ventricle to clear the blood in the pulmonary vasculature. Then, the left lower lung lobe was excised, fixed in $4 \%$ paraformaldehyde for $24 \mathrm{~h}$, embedded in paraffin, cut into $5 \mu \mathrm{m}$ sections on a Leica model 2165 rotary microtome (Leica, Nussloch, Germany), and stained with H\&E. For each animal, five fields were measured randomly from the H\&E staining at a magnification of $\times 200$.

\section{Wet/Dry lung weight ratio measurement}

The right upper lung lobes from each group were excised at the end of the experiment and weighed to determine the wet lung weight. The tissues were then placed in a metal bath and incubated at a temperature of $80^{\circ} \mathrm{C}$ for $24 \mathrm{~h}$ before being weighed again to give the final dry weight. The presence of edema was determined by calculating the pulmonary W/D ratio ${ }^{51,52}$.

\section{Measurement of bronchoalveolar epithelial permeability}

To measure the bronchoalveolar epithelial permeability, a fluorescein isothiocyanate-conjugated dextran 4000 (FD4, Sigma-Aldrich, Bornem, Belgium) solution in PBS $(25 \mathrm{mg} / \mathrm{ml} ; 10 \mathrm{mg} / \mathrm{kg}$ ) was injected intraperitoneally. Four hours later, after anesthesia and pulmonary perfusion, the fluorescence intensity of FD4 in homogenized lung tissue was measured using a fluorescence plate reader (Biotek synergy) at an excitation wavelength of $485 \mathrm{~nm}$ and an emission wavelength of $528 \mathrm{~nm}^{53,54}$.

\section{Transmission electron microscopy}

After dissection of the lung, the specimens were fixed overnight in $2.5 \%$ glutaraldehyde at $4{ }^{\circ} \mathrm{C}$. Then, the tissues were washed, fixed, dehydrated, and embedded. Random 60-nm transverse sections were collected on single copper slot grids coated with parlodion, stained with uranyl acetate and, lead citrate and observed with an FEI Tecnai G2 Spirit Bio TWIN transmission electron microscope.

\section{Flow cytometry}

To prepare single cells from whole lungs, lung tissue was ground in PBS containing 1\% BSA. Freed cells were washed with PBS containing 1\% BSA and filtered using 70-mm nylon mesh. Cells $\left(10^{6} / 100 \mu \mathrm{l}\right)$ were blocked with $1 \mu \mathrm{l}$ of purified anti-mouse CD16/CD32 (14-0161-85, Thermo) (FcR block) antibody for $10 \mathrm{~min}$ on ice to prevent 
Table 1 Primers used in the study

\begin{tabular}{lll}
\hline Gene ID & Forward primer & Reverse primer \\
\hline GAPDH & AGGTCGGTTGAACGGATTTG & TGTAGACCATGTAGTTGA GGTCA \\
TNF-a & TCTCATCAGTTCTATGGCCC & GGGAGTAGACAAGGTACAAC \\
IL-1 $\beta$ & AGCTTCAAATCTCGCAGCAG & TCTCCACAGCCACAATGAGT \\
IL-6 & GGCGGATCGGATGTTGTGAT & GGACCCCAGACAATCGGTTG \\
KC & CAAGGCTGGTCCATGCTCC & TGCTATCACTTCCTTTCTGTTGC \\
CXCL2 & CCAACCACCAGGCTACAGG & GCGTCACACTCAAGCTCTG \\
CXCL5 & GTTCCATCTCGCCATTCATGC GCGCTATGACTGAGGAAGG \\
CCL3 & TTCTCTGTACCATGACACTCTGC & CGTGGAATCTTCCGGCTGTAG \\
CCL4 & TTCCTGCTGTTTCTCTTACACCT & CTGTCTGCCTCTTTTGGTCAG \\
MCP-1 & GTTGGCCTCAGCCAGATGCA & AGCGTACTCATTGGGATCATCTTG \\
\hline
\end{tabular}

nonspecific staining and then stained in the dark with a combination of anti-mouse CD45-FITC (MCD4501, Thermo), anti-mouse Ly6G-PE (12-5931-85, Thermo), and anti-mouse F4/80 antigen APC (17-4801-82, Thermo) at room temperature for $1 \mathrm{~h}$. The stained cells were washed three times with ice-cold PBS and analyzed with a BD FACS ARIA II SORP (Special Order Research Product) flow cytometer with FlowJo_V10 software.

\section{RNA isolation and quantitative real-time PCR}

Total RNA was obtained from fresh lung tissue with a TRIzol reagent kit (Life Technologies) and reversetranscribed into cDNA with a reverse-transcription kit (Abm, Zhenjiang, China) according to the manufacturer's instructions. TNF- $\alpha$, IL-1 $\beta$, IL-6, KC, CXCL2, CXCL5, CCL3, CCL4, MCP-1, and GAPDH mRNA expression was detected by a StepOnePlus Real-Time PCR System (ABI, USA). The primer sequences used for real-time PCR were designed by referring to PrimerBank (https://pga. mgh.harvard.edu/primerbank). The primers for those genes are shown in Table 1. Relative levels were determined using the $2^{-\Delta \Delta C t}$ method, and GAPDH was used as the internal control ${ }^{55}$. Each sample was run in triplicate, and the results were representative of at least three independent experiments.

\section{Enzyme-linked immunosorbent assay}

To measure the secretion of CXCL1/KC and MCP-1 in mouse lung tissue, ELISAs were performed according to the manufacturer's protocols using the appropriate ELISA kits (Mouse CXCL1/KC DuoSet ELISA, DY453-05, R\&D Systems, and Mouse MCP-1 ELISA MAX ${ }^{\mathrm{rm}}$ Deluxe, 432704, BioLegend). Then, the optical density was spectrophotometrically measured at $450 \mathrm{~nm}$ using a microplate reader (Biotek Instruments, Inc., USA).

\section{Protein extraction and western blotting}

Total proteins were extracted from lung tissue by lysis with RIPA buffer containing protease and phosphatase inhibitor cocktails (Beyotime, Shanghai, China). For neutrophils, the culture supernatant was solubilized in $5 \times$ concentrated sodium dodecyl sulfate-polyacrylamide gel electrophoresis (SDS-PAGE) sample buffer and boiled at $100{ }^{\circ} \mathrm{C}$ for $5 \mathrm{~min}$. Protein concentrations were determined by the BCA method. Equal amounts of proteins were used for SDS-PAGE. After gel electrophoresis, the proteins were transferred into polyvinylidene difluoride membranes (Millipore, Billerica, USA). The membranes were blocked for $1 \mathrm{~h}$ in $5 \%$ skim milk at room temperature and incubated at $4{ }^{\circ} \mathrm{C}$ overnight with the following primary antibodies: anti-histone 3 (citrulline $\mathrm{R} 2+\mathrm{R} 8+\mathrm{R} 17$, ab5103, Abcam), anti-ZO-1 (ab216880), anti-occludin (ab216327), anti-E-cadherin (ab76055), and antiGAPDH (5174s, Cell Signaling Technology). The membranes were then washed three times with TBST and followed by an incubation with horseradish peroxidase (HRP)-conjugated goat anti-rabbit IgG (EarthOx Life Sciences, CA, USA) or goat anti-mouse IgG $(\mathrm{H}+\mathrm{L}) \mathrm{HRP}$ (s0002, Affinity Biosciences) for $1 \mathrm{~h}$ at room temperature. GAPDH was used as the internal control. The antibody-antigen complexes were detected with Immobilon Western Chemiluminescent HRP Substrate (Millipore, MA, USA) and visualized using the G:Box gel doc system (Syngene, UK).

\section{Neutrophil isolation}

Mouse bone marrow-derived neutrophils were isolated using a neutrophil isolation kit by following a protocol provided by the manufacturer (130-097-658, Miltenyi Biotec). The isolation procedure was conducted at $4{ }^{\circ} \mathrm{C}$ unless otherwise specified. In brief, mice were anesthetized 
and the animal surface was sprayed with $70 \%$ ethanol. The muscles were removed from both legs and the femur was separated from the tibia at the knee joint. The bone marrow cells were flushed using a sterile syringe filled with PBS containing $0.5 \%$ endotoxin-free BSA and $2 \mathrm{mM}$ EDTA (magnetic-activated cell-sorting, MACS) and collected into a $50 \mathrm{ml}$ conical tube through a $70 \mu \mathrm{m}$ cell strainer. The bone marrow cells were collected by centrifugation at $500 \times g$ for $5 \mathrm{~min}$ at $4{ }^{\circ} \mathrm{C}$. The cell pellet was resuspended in $1 \mathrm{ml}$ of RBC lysis buffer for $\sim 5 \mathrm{~min}$. The cell suspension was washed and centrifuged to collect the cells. The cell pellet was resuspended in $200 \mu \mathrm{l}$ MACS buffer and $50 \mu \mathrm{l}$ neutrophil biotin-antibody cocktail was added. Then, the cell suspension was mixed thoroughly and incubated for $10 \mathrm{~min}$ in the refrigerator at $4{ }^{\circ} \mathrm{C}$. The cell suspension was washed and centrifuged to collect the cells. The cell pellet was resuspended in $400 \mu \mathrm{l}$ MACS buffer and $100 \mu \mathrm{l}$ antibiotin microbeads was added. The cell suspension was mixed well and incubated for $15 \mathrm{~min}$ in the refrigerator at $4{ }^{\circ} \mathrm{C}$. The cell suspension was washed and centrifuged to collect the cells. The cell pellet was resuspended in $500 \mu \mathrm{l}$ of MACS buffer. The cells were subsequently loaded onto a MACS buffer equilibrated LS column (Miltenyi Biotec) and washed LS column three times with $3 \mathrm{ml}$ of MACS buffer. The cells through the LS column were harvested and allowed to warm up to room temperature in RPMI medium until they were used.

\section{NE-DNA quantification}

The level of NE-DNA in the culture supernatant of neutrophils was measured by ELISA as described previously but with minor modifications ${ }^{56}$. In brief, a 96-well ELISA microplate was coated with ELA2 antibody (Proteintech, 17943-1-AP, $1: 2000)$ and incubated at $4{ }^{\circ} \mathrm{C}$ overnight. After washing three times, the uncoated sites were blocked with $1 \%$ bovine serum albumin (BSA) in phosphate-buffered saline (PBS) at $37^{\circ} \mathrm{C}$ for 1 hour. The microwells were again washed, and the samples were added to individual wells. The plate was incubated at $4{ }^{\circ} \mathrm{C}$ overnight. After washing, HRP-conjugated anti-DNA antibody (D5425-3-200, 1:100) was added to each well, and the plate was incubated at room temperature for $2 \mathrm{~h}$. The plate was again washed, and TMB substrate was added. Absorbance was measured at $450 \mathrm{~nm}$ (end point) with a microplate reader (Biotek synergy) after the addition of the $2 \mathrm{~N} \mathrm{H} 2 \mathrm{SO} 4$ stop solution. Background absorbance values of the medium were subtracted.

\section{Flow cytometric assay for direct quantification of neutrophil extracellular traps}

A flow cytometric assay was performed for NETs as described previously ${ }^{57}$. Briefly, neutrophils $\left(1 \times 10^{6}\right)$ were seeded on a 24-well cell culture plate and incubated for $1 \mathrm{~h}$ in a $\mathrm{CO}_{2}$ incubator at $37^{\circ} \mathrm{C}$. Then, the cells were either left unstimulated or stimulated with $100 \mathrm{nM}$ PMA or $1 \mu \mathrm{g} / \mathrm{ml} \mathrm{VP1} \mathrm{for} 4 \mathrm{~h}$. After the incubation, neutrophils were collected, fixed in $2 \%$ paraformaldehyde, blocked for 30 min with $5 \%$ goat sera without a permeabilization step, and incubated sequentially with the primary anti-histone $\mathrm{H} 3$ antibody (1:500, citrulline 2,8,17, ab5103, Abcam) at $4^{\circ} \mathrm{C}$ for $1 \mathrm{~h}$ and with goat anti-rabbit IgG $(\mathrm{H}+\mathrm{L})$ CrossAdsorbed Secondary Antibody, Alexa Fluor 555 (1:500, A21428, Thermo) at $37^{\circ} \mathrm{C}$ for $1 \mathrm{~h}$ in the dark. Each incubation was followed by a wash with $2 \%$ BSA in PBS and centrifugation at $4{ }^{\circ} \mathrm{C}$ for $5 \mathrm{~min}(2000 \mathrm{rpm})$. Samples were then analyzed by flow cytometry.

In a parallel experiment, prior to stimulation with PMA and VP1, neutrophils were pretreated with specific inhibitors for $30 \mathrm{~min}$, including the protein arginine deiminase 4 (PAD4) inhibitor $\mathrm{Cl}$-amidine $(200 \mu \mathrm{M}$, Selleck) and the NADPH oxidase inhibitor DPI chloride $(10 \mu \mathrm{M}$, Thermo).

\section{Confocal microscopy}

Neutrophils $\left(1 \times 10^{6}\right)$ were seeded on a sterile round glass cover slip that was placed in a 24-well cell culture plate. As described above in the flow cytometric assay for NETs, $100 \mu \mathrm{M}$ PMA or $1 \mu \mathrm{g} / \mathrm{ml}$ VP1 was added. After $4 \mathrm{~h}$ of incubation, the glass cover slips with the attached cells were carefully removed from a 24-well culture plate and fixed with ice-cold acetone. Then, samples were blocked with $5 \%$ goat sera and stained overnight with a rabbit polyclonal antibody to histone 3 (citrulline $\mathrm{R} 2+\mathrm{R} 8+$ R17) (1:300, ab5103, Abcam) and with a mouse anti-MPO antibody (1:300, ab90810, Abcam). The samples were washed in PBST and further stained with an Alexa Fluor ${ }^{\circledR}$ 555 goat anti-rabbit antibody (1:500, Life Technologies, USA) and an Alexa Fluor ${ }^{\circledR} 647$ goat anti-mouse antibody (1:1000, Life Technologies, USA). Nuclei in the samples were stained with 4'6-diamidino-2-phenylindole. Images were captured by Zeiss LSM7 confocal fluorescence microscopes using the appropriate lenses and filters.

For experiments in vivo, the left lower lung of mice that were or were not treated with VP1 was carefully harvested, frozen in Tissue-Tek OCT media and prepared into $7-\mu \mathrm{m}$-thick slices. The fixation, blocking, antibody incubation, and image acquisition of these sections were performed as described at the cell level.

\section{Transepithelial electrical resistance (TEER) measurement}

The TEER of the MLE-12 cell monolayer was measured using a Millicell ${ }^{\circledR}$ ERS-2 Electrical Resistance System. To eliminate the influence of temperature, the measurements were performed within $5 \mathrm{~min}$ after taking the culture plates out of the incubator. During this time, the samples did not show any reading drift. Before measurement, the electrodes were equilibrated and sterilized according to the manufacturer's recommendations. Two hundred microliters of 
culture medium were added to the upper compartment of the cell culture system. The ohmic resistance of a blank (culture insert without cells) was measured in parallel. To obtain the sample resistance, the blank value was subtracted from the total resistance of the sample. The final unit area resistance $\left(\Omega \mathrm{cm}^{2}\right)$ was calculated by multiplying the sample resistance by the effective area of the membrane $\left(0.3 \mathrm{~cm}^{2}\right.$ for 24-well Millicell inserts).

\section{Live-cell imaging technique}

Neutrophils $\left(5 \times 10^{4} /\right.$ well $)$ were seeded onto a 96 -well cell culture plate and treated with culture medium or VP1. Next, $1 \mu$ M SYTOX Orange (S11368, Thermo) was added before transferring the 96-well cell culture plate into the temperature- and $\mathrm{CO}_{2}$-controlled $\left(37^{\circ} \mathrm{C}, 5 \%\right.$ $\mathrm{CO}_{2}$ ) environment of a Zeiss Cell Discoverer 7 microscope system. Live-cell phase-gradient contrast images of the individual field regions inside each well were automatically acquired using the ZEN Blue 2.3 software. Representative figure images were selected and additional image postprocessing steps (contrast adjustment, field selection, and scale bar addition) were performed in the Zeiss ZEN software.

\section{Statistics}

Statistical analyses were performed using GraphPad Prism 7.0 software. Details of the individual tests are included in the figure legends. In general, data were tested for a normal distribution by the Kolmogorov-Smirnov normality test and were analyzed accordingly by an unpaired two-tailed $t$-test or the Mann-Whitney $U$-test. Data are presented as the mean \pm SEM. In all cases, $P$ values $<0.05$ were considered significant.

\section{Acknowledgements}

This study was supported by the National Natural Science Foundation of China, grant 31470889 and 81671563.

\section{Author details \\ 'Department of Immunology, Nanjing Medical University, 210016 Nanjing, China. ${ }^{2} \mathrm{NHC}$ Key Lab of Antibody Technique, Nanjing Medical University, 210016 Nanjing, China. ${ }^{3}$ Department of Respiratory and Critical Care Medicine, The First Affiliated Hospital of Nanjing Medical University, 210029 Nanjing, China. ${ }^{4}$ The Laboratory Center for Basic Medical Sciences, Nanjing Medical University, 210016 Nanjing, China. ${ }^{5}$ Department of Infectious Disease, Nanjing Medical University Nanjing First Hospital, 210006 Nanjing, China. ${ }^{6}$ Analysis center, Nanjing Medical University, 210016 Nanjing, China. ${ }^{7}$ State Key Laboratory of Reproductive Medicine, Nanjing Medical University, 210016 Nanjing, China}

\section{Authors' contributions}

Conceived and designed research: M.S.Z and X.J. Performed experiments: N.W., X.F.Y., J.D.S., Z.X.S., Q.Y.M., Z.X.W., Z.Q.C., Z.B.W. and F.H. Analyzed the data: N.W., X.F.Y., H.J.W., L.F.Z., M.S.Z. and J.X. Interpreted results of experiments: N.W., X.F.Y., J.D.S. and M.S.Z., Prepared figures: N.W., X.F.Y. and J.D.S. Drafted the manuscript: N.W., X.F.Y. and J.D.S. Edited and revised the manuscript: M.S.Z. and J.X. All the authors approved final version of the manuscript.

\section{Conflict of interest}

The authors declare that they have no conflict of interest

\section{Publisher's note}

Springer Nature remains neutral with regard to jurisdictional claims in published maps and institutional affiliations.

The online version of this article (https://doi.org/10.1038/s41420-019-0193-3) contains supplementary material, which is available to authorized users.

Received: 30 April 2019 Revised: 18 May 2019 Accepted: 25 May 2019 Published online: 04 July 2019

\section{References}

1. $\mathrm{Xu}$, J. et al. EV71: an emerging infectious disease vaccine target in the Far East? Vaccine 28, 3516-3521 (2010).

2. Cox, J. A., Hiscox, J. A., Solomon, T., Ooi, M. H. \& Ng, L. F. P. Immunopathogenesis and virus-host interactions of enterovirus 71 in patients with hand, foot and mouth disease. Front. Microbiol. 8, 2249 (2017).

3. Jin, Y. et al. Pulmonary edema following central nervous system lesions induced by a non-mouse-adapted EV71 strain in neonatal BALB/C mice. Virol. J. 14, 243 (2017).

4. Yu, P. et al. Distribution of enterovirus 71 RNA in inflammatory cells infiltrating different tissues in fatal cases of hand, foot, and mouth disease. Arch. Virol. 160, 81-90 (2015).

5. Yu, P. et al. Histopathological features and distribution of EV71 antigens and SCARB2 in human fatal cases and a mouse model of enterovirus 71 infection. Virus Res. 189, 121-132 (2014).

6. Tee, K. K. et al. Evolutionary genetics of human enterovirus 71: origin, population dynamics, natural selection, and seasonal periodicity of the VP1 gene. J. Virol. 84, 3339-3350 (2010).

7. Huang, S. W., Wang, Y. F., Yu, C. K., Su, I. J. \& Wang, J. R. Mutations in VP2 and VP1 capsid proteins increase infectivity and mouse lethality of enterovirus 71 by virus binding and RNA accumulation enhancement. Virology 422, 132-143 (2012).

8. Brinkmann, V. et al. Neutrophil extracellular traps kill bacteria. Science 303, 1532-1535 (2004).

9. Papayannopoulos, V., Metzler, K. D., Hakkim, A. \& Zychlinsky, A. Neutrophil elastase and myeloperoxidase regulate the formation of neutrophil extracellular traps. J. Cell. Biol. 191, 677-691 (2010).

10. Kaplan, M. J. \& Radic, M. Neutrophil extracellular traps: double-edged swords of innate immunity. J. Immunol. 189, 2689-2695 (2012).

11. Raftery, M. J. et al. beta2 integrin mediates hantavirus-induced release of neutrophil extracellular traps. J. Exp. Med. 211, 1485-1497 (2014).

12. Saitoh, T. et al. Neutrophil extracellular traps mediate a host defense response to human immunodeficiency virus-1. Cell Host Microbe 12, 109-116 (2012).

13. $\mathrm{Ng}, \mathrm{H}$. H. et al. Doxycycline treatment attenuates acute lung injury in mice infected with virulent influenza H3N2 virus: involvement of matrix metalloproteinases. Exp. Mol. Pathol. 92, 287-295 (2012).

14. Saffarzadeh, M. et al. Neutrophil extracellular traps directly induce epithelial and endothelial cell death: a predominant role of histones. PloS ONE 7, e32366 (2012).

15. Narasaraju, T. et al. Excessive neutrophils and neutrophil extracellular traps contribute to acute lung injury of influenza pneumonitis. Am. J. Pathol. 179, 199-210 (2011)

16. Porto, B. N. \& Stein, R. T. Neutrophil extracellular traps in pulmonary diseases: too much of a good thing? Front. Immunol. 7, 311 (2016).

17. Cavanaugh, K. J. Jr., Oswari, J. \& Margulies, S. S. Role of stretch on tight junction structure in alveolar epithelial cells. Am. J. Respir. Cell Mol. Biol. 25, 584-591 (2001).

18. Liu, M., Gu, C. \& Wang, Y. Upregulation of the tight junction protein occludin: effects on ventilation-induced lung injury and mechanisms of action. BMC Pulm. Med. 14, 94 (2014)

19. Zhao, T., Liu, M., Gu, C., Wang, X. \& Wang, Y. Activation of c-Src tyrosine kinase mediated the degradation of occludin in ventilator-induced lung injury. Respir. Res. 15, 158 (2014).

20. Post, S. et al. Characterization of a lung epithelium specific E-cadherin knockout model: implications for obstructive lung pathology. Sci. Rep. 8, 13275 (2018).

21. Du, N. et al. Cell surface vimentin is an attachment receptor for enterovirus 71 J. Virol. 88, 5816-5833 (2014). 
22. De Filippo, K. et al. Mast cell and macrophage chemokines CXCL1/CXCL2 control the early stage of neutrophil recruitment during tissue inflammation. Blood 121, 4930-4937 (2013).

23. Lin, M., Carlson, E., Diaconu, E. \& Pearlman, E. CXCL1/KC and CXCL5/LIX are selectively produced by corneal fibroblasts and mediate neutrophil infiltration to the corneal stroma in LPS keratitis. J. Leukoc. Biol. 81, 786-792 (2007).

24. Ritzman, A. M. et al. The chemokine receptor CXCR2 ligand KC (CXCL1) mediates neutrophil recruitment and is critical for development of experimental Lyme arthritis and carditis. Infect. Immun. 78, 4593-4600 (2010).

25. Owen, J. L. \& Mohamadzadeh, M. Macrophages and chemokines as mediators of angiogenesis. Front. Physiol. 4, 159 (2013).

26. Patterson, A. M. et al. Differential binding of chemokines to macrophages and neutrophils in the human inflamed synovium. Arthritis Res. 4, 209-214 (2002).

27. Sorensen, O. E. \& Borregaard, N. Neutrophil extracellular traps - the dark side of neutrophils. J. Clin. Investig. 126, 1612-1620 (2016).

28. Farley, K., Stolley, J. M., Zhao, P., Cooley, J. \& Remold-O'Donnell, E. A serpinB1 regulatory mechanism is essential for restricting neutrophil extracellular trap generation. J. Immunol. 189, 4574-4581 (2012).

29. Page, M. J., Bester, J. \& Pretorius, E. The inflammatory effects of TNF-alpha and complement component 3 on coagulation. Sci. Rep. 8, 1812 (2018).

30. Brinkmann, V., Laube, B., Abu Abed, U., Goosmann, C. \& Zychlinsky, A. Neutrophil extracellular traps: how to generate and visualize them. J. Visual. Exp. https://doi.org/10.3791/1724 (2010).

31. Ralston, K. S. et al. Trogocytosis by Entamoeba histolytica contributes to cell killing and tissue invasion. Nature 508, 526-530 (2014).

32. Neubert, E. et al. Chromatin swelling drives neutrophil extracellular trap release. Nat. Commun. 9, 3767 (2018).

33. Li, P. et al. PAD4 is essential for antibacterial innate immunity mediated by neutrophil extracellular traps. J. Exp. Med. 207, 1853-1862 (2010).

34. Wang, Y. et al. Histone hypercitrullination mediates chromatin decondensation and neutrophil extracellular trap formation. J. Cell Biol. 184, 205-213 (2009).

35. Martinod, K. et al. Neutrophil histone modification by peptidylarginine deiminase 4 is critical for deep vein thrombosis in mice. Proc. Natl Acad. Sci. USA 110, 8674-8679 (2013).

36. Fuchs, T. A. et al. Novel cell death program leads to neutrophil extracellular traps. J. Cell Biol. 176, 231-241 (2007).

37. Campbell, A. M., Kashgarian, M. \& Shlomchik, M. J. NADPH oxidase inhibits the pathogenesis of systemic lupus erythematosus. Sci. Transl. Med. 4, 157ra141 (2012).

38. Knight, J. S. et al. Peptidylarginine deiminase inhibition disrupts NET formation and protects against kidney, skin and vascular disease in lupus-prone MRL/pr mice. Ann. Rheum. Dis. 74, 2199-2206 (2015).

39. Knight, J. S. et al. Peptidylarginine deiminase inhibition reduces vascular damage and modulates innate immune responses in murine models of atherosclerosis. Circ. Res. 114, 947-956 (2014).
40. Hosseinzadeh, A., Messer, P. K. \& Urban, C. F. Stable redox-cycling nitroxide tempol inhibits NET formation. Front. Immunol. 3, 391 (2012).

41. Kraaij, T. et al. A novel method for high-throughput detection and quantification of neutrophil extracellular traps reveals ROS-independent NET release with immune complexes. Autoimmun. Rev. 15, 577-584 (2016).

42. Eneling, $\mathrm{K}$. et al. Salt-inducible kinase 1 regulates E-cadherin expression and intercellular junction stability. FASEB J. 26, 3230-3239 (2012).

43. Bachler, G. et al. Translocation of gold nanoparticles across the lung epithelial tissue barrier: combining in vitro and in silico methods to substitute in vivo experiments. Part. Fibre Toxicol. 12, 18 (2015).

44. Gilles, C. et al. Vimentin contributes to human mammary epithelial cell migration. J. Cell Sci. 112(Pt 24), 4615-4625 (1999).

45. Wang, S. M. et al. Pathogenesis of enterovirus 71 brainstem encephalitis in pediatric patients: roles of cytokines and cellular immune activation in patients with pulmonary edema. J. Infect. Dis. 188, 564-570 (2003).

46. Lin, T. Y., Hsia, S. H., Huang, Y. C., Wu, C. T. \& Chang, L. Y. Proinflammatory cytokine reactions in enterovirus 71 infections of the central nervous system. Clin. Infect. Dis. 36, 269-274 (2003).

47. Jin, Y. et al. Mast cells contribute to enterovirus 71 infection-induced pulmonary edema in neonatal mice. Lab. Investig. 98, 1039-1051 (2018).

48. Schonrich, G. \& Raftery, M. J. Neutrophil extracellular traps go viral. Front Immunol. 7, 366 (2016).

49. Herrero, R. et al. Fas activation alters tight junction proteins in acute lung injury. Thorax 74, 69-82 (2018).

50. Albrengues, J. et al. Neutrophil extracellular traps produced during inflammation awaken dormant cancer cells in mice. Science 361, eaao4227 (2018).

51. Larche, J. et al. Inhibition of mitochondrial permeability transition prevents sepsis-induced myocardial dysfunction and mortality. J. Am. Coll. Cardiol. 48, 377-385 (2006)

52. Folz, R. J., Abushamaa, A. M. \& Suliman, H. B. Extracellular superoxide dismutase in the airways of transgenic mice reduces inflammation and attenuates lung toxicity following hyperoxia. J. Clin. Investig. 103, 1055-1066 (1999).

53. Han, X., Fink, M. P., Uchiyama, T., Yang, R. \& Delude, R. L. Increased iNOS activity is essential for pulmonary epithelial tight junction dysfunction in endotoxemic mice. Am. J. Physiol. Lung Cell. Mol. Physiol. 286, L259-L267 (2004).

54. You, K. et al. Hyperoxia disrupts pulmonary epithelial barrier in newborn rats via the deterioration of occludin and ZO-1. Respir. Res 13, 36 (2012).

55. Livak, K. J. \& Schmittgen, T. D. Analysis of relative gene expression data using real-time quantitative PCR and the 2(-Delta Delta C(T)) Method. Methods 25, 402-408 (2001).

56. Kano, H., Aminul Huq, M., Tsuda, M., Noguchi, H. \& Takeyama, N. Sandwich ELISA for circulating myeloperoxidase- and neutrophil elastase-DNA complexes released from neutrophil extracellular traps. Adv. Tech. Biol. Med. 05, https://doi.org/10.4172/2379-1764.1000196 (2017).

57. Gavillet, M. et al. Flow cytometric assay for direct quantification of neutrophil extracellular traps in blood samples. Am. J. Hematol. 90, 1155-1158 (2015). 